\title{
ON THE GENERALIZED BLASCHKE CONDITION
}

\author{
BY
}

P. S. CHEE

\begin{abstract}
In an earlier paper, the author has shown that the generalized Blaschke condition is satisfied by bounded holomorphic functions in the polydisc or the ball in $C^{N}$. By essentially the same method, it is shown in the present paper that the same condition is satisfied by larger classes of functions, the Nevanlinna classes.
\end{abstract}

1. Introduction. Let $\Omega$ denote the unit polydisc $U^{n+1}$ or the unit ball $B_{n+1}$ in the space $C^{n+1}$ of $(n+1)$ complex variables. Let $f \not \equiv 0$ be a bounded holomorphic function in $\Omega$ and let $\mu(z)$ be its zero-multiplicity at $z \in \Omega$. Let $H_{k}$ denote the Hausdorff $k$-measure in $C^{n+1}$. $\left(H_{2 n+2}\right.$ is equal to the Lebesgue measure on $C^{n+1}$.) It was shown in [1] that the following Blaschke condition holds for $f$ :

$$
\int_{0}^{1} d r \int_{\bar{\Omega}(r)} \mu(z) d H_{2 n}(z)<\infty,
$$

where $\Omega(r)$ is the polydisc or ball of radius $r$. This generalizes the well-known Blaschke condition for bounded holomorphic functions in the unit disc $U$. Professor P. Ahern raised the question if the condition (1.1) was also satisfied by functions in the Hardy class $H^{2}(\Omega)$. The purpose of this paper is to answer this in the affirmative. In fact we shall show that the Blaschke condition is satisfied by an even larger class of functions, namely, the Nevanlinna class $N(\Omega)$. This again generalizes the one variable result.

For convenience, we shall denote by $\partial \Omega$ the distinguished boundary $T^{n+1}$ of $U^{n+1}$ when $\Omega=U^{n+1}$ and the $(2 n-1)$ dimensional sphere $S^{2 n-1}$ when $\Omega=B_{n+1}$. For $f$ holomorphic in $\Omega$, we define

$$
\|f\|_{0}=\sup _{0 \leqq r<1} \int_{\partial \Omega} \log ^{+}|f(r w)| d m(w)
$$

where $m$ is the Lebesgue measure on $\partial \Omega$, normalized so that $m(\partial \Omega)=1$. (We shall use $m$ to denote the Lebesgue measure. The dimension will be clear from the context.) The Nevanlinna class on $\Omega$ is defined as

$$
N(\Omega)=\left\{f \text { holomorphic in } \Omega:\|f\|_{0}<\infty\right\} .
$$

Our main result is

Received by the editors November 20, 1969.

Key words and phrases. Polydiscs, balls, generalized Blaschke condition, Nevanlinna classes, radial limits, pluri-subharmonic functions, integral formula on balls.

Copyright (C) 1970, American Mathematical Society 
THEOREM 1.1. Let $f \in N(\Omega), f \not \equiv 0$, and let $\mu$ be its zero-multiplicity. Then $f$ satisfies the Blaschke condition (1.1).

Our proof follows that of [1]. We shall use the notation and results established there.

I wish to thank Professor Ahern for raising the question.

2. Scheme of proof of Theorem 1.1. The points of $C^{n+1}$ will be denoted by $z=\left(z_{1}, \ldots, z_{n}, z_{n+1}\right)$. For $1 \leqq j \leqq n+1$, let $\pi_{j}: C^{n+1} \rightarrow C^{n+1}$ be the projection $\pi_{j}(z)=\left(z_{1}, \ldots, z_{j-1}, 0, z_{j+1}, \ldots, z_{n+1}\right) \equiv \hat{z}_{j}$. Let $V_{r}=\{z \in \bar{\Omega}(r): f(z)=0\}$ and

$$
F\left(r, \hat{z}_{j}\right)=\int_{V_{r} \cap_{\pi_{j}}^{-1}\left(\hat{(}_{j}\right)} \mu(z) d H_{0}\left(z_{j}\right) .
$$

By formulas (19) and (20) of $\S 6$ in [1], we have

$$
\int_{0}^{1} d r \int_{\bar{\Omega}(r)} \mu(z) d H_{2 n}(z)=\sum_{j=1}^{n+1} \int_{\pi, \Omega} d H_{2 n}\left(\hat{z}_{j}\right) \int_{0}^{1} F\left(r, \hat{z}_{j}\right) d r .
$$

For each $j, 1 \leqq j \leqq n+1$, define $f_{\hat{z},}$ by $f_{\hat{z}_{j}}\left(z_{j}\right)=f(z)$. Let $a=1$ if $\Omega=U^{n+1}$, and $a=\left(1-\left\|\hat{z}_{j}\right\|^{2}\right)^{1 / 2}$ if $\Omega=B_{n+1}$. For $w \in T$, let $f_{\hat{z}_{j}}^{*}(a w)=\lim _{r \rightarrow 1} f_{\hat{z}_{j}}(a r w)$. Then Jensen's formula (equation (2) in [1]; see also [3, §3.61]) gives

$$
\int_{0}^{1} F\left(r, \hat{z}_{j}\right) d r \leqq \int_{T} \log \left|f_{z_{j}}^{*}(a w)\right| d m_{1}(w)-\log \left|f\left(\hat{z}_{j}\right)\right|,
$$

where $m_{1}$ is the normalized Lebesgue measure on $T$. Hence

$$
\begin{aligned}
\int_{0}^{1} d r \int_{\bar{\Omega}(r)} \mu(z) d H_{2 n}(z) \leqq & \sum_{j=1}^{n+1} \int_{\pi_{j} \Omega} d H_{2 n}\left(\hat{z}_{j}\right) \int_{T} \log \left|f_{z_{j}}^{*}(a w)\right| d m_{1}(w) \\
& -\sum_{j=1}^{n+1} \int_{\pi_{j} \Omega} \log \left|f\left(\hat{z}_{j}\right)\right| d H_{2 n}\left(\hat{z}_{j}\right) .
\end{aligned}
$$

We may assume without loss of generality that $f\left(\hat{z}_{j}\right) \neq 0$ for all $j$ (see $[1, \S 6]$ ). We shall show in the following sections that, under this assumption, each integral on the right is finite (Lemmas 3.3 and 3.4). This will imply that the Blaschke condition (1.1) holds for $f$.

3. Integrals of functions in $N(\Omega)$. Let $f$ be a holomorphic function in $\Omega$. For $w \in \partial \Omega$, let $f^{*}(w)=\lim _{r \rightarrow 1} f(r w)$ when the limit exists.

THEOREM 3.1. If $f \in N(\Omega), f \not \equiv$, then $f^{*}(w)$ exists for almost all $w \in \partial \Omega$. Furthermore,

$$
\log \left|f^{*}\right| \in L^{1}(\partial \Omega)
$$

and

$$
\int_{\partial \Omega} \log \left|f^{*}(w)\right| d m(w) \leqq\|f\|_{0}
$$


The proof for $\Omega=U^{n+1}$ is given in [2, p. 46]; the proof for $\Omega=B_{n+1}$ is exactly similar.

LeMma 3.2. If $f \in N(\Omega), f \not \equiv 0$, then $\log |f| \in L^{1}(\Omega)$ and $\log ^{+}|f| \in L^{1}(\Omega)$.

Proof. Since $\log |f|$ and $\log ^{+}|f|$ are pluri-subharmonic in $\Omega$, Theorem 5.2 of [1] gives

$$
\int_{\Omega} \log ^{+}|f(z)| d m(z) \geqq \int_{\Omega} \log |f(z)| d m(z)>-\infty .
$$

Since $\log |f|$ and $\log ^{+}|f|$ are $(n+1)$-subharmonic (see [2, §3.2]), the following integrals are nondecreasing functions of each $r_{j}$. So for $\Omega=U^{n+1}$,

$$
\begin{aligned}
\int_{T^{n+1}} \log \mid f\left(r_{1} w_{1}, \ldots,\right. & \left.r_{n+1} w_{n+1}\right) \mid d m_{1}\left(w_{1}\right) \cdots d m_{1}\left(w_{n+1}\right) \\
& \leqq \int_{T^{n+1}} \log ^{+}\left|f\left(r_{1} w_{1}, \ldots, r_{n+1} w_{n+1}\right)\right| d m_{1}\left(w_{1}\right) \cdots d m_{1}\left(w_{n+1}\right) \\
& \leqq\|f\|_{0} \quad \text { for } 0 \leqq r_{j}<1,1 \leqq j \leqq n+1 .
\end{aligned}
$$

Multiplying by $r_{1} d r_{1} \cdots r_{n+1} d r_{n+1}$ and integrating from 0 to 1 for each $r_{j}$, we get

$$
\int_{U^{n+1}} \log |f(z)| d m(z) \leqq \int_{U^{n+1}} \log ^{+}|f(z)| d m(z) \leqq 2^{-n-1}\|f\|_{0} .
$$

Similarly for $\Omega=B_{n+1}$, we have by the subharmonicity of $\log |f|$ and $\log ^{+}|f|$,

$$
\int_{\partial_{B_{n}+1}} \log |f(r w)| d m(w) \leqq \int_{\partial_{B_{n+1}}} \log ^{+}|f(r w)| d m(w) \leqq\|f\|_{0}
$$

for $0<r<1$. Multiplying by $r^{2 n+1} d r$ and integrating from 0 to 1 , we get

$$
\int_{B_{n+1}} \log |f(z)| d m(z) \leqq \int_{B_{n+1}} \log ^{+}|f(z)| d m(z) \leqq \frac{\|f\|_{0}}{2 n+2}
$$

The lemma follows from (3.1), (3.2) and (3.3).

LEMMA 3.3. Let $f \in N(\Omega)$ and $1 \leqq j \leqq n+1$. If $f\left(\hat{z}_{j}\right) \not \equiv 0$, then $\log |f(\hat{z})| \in L^{1}\left(\pi_{j} \Omega\right)$.

Proof. Let $a$ be as defined in $\S 2$ above and $U(a)$ the disc of radius $a$. By the $(n+1)$-subharmonicity of $\log ^{+}|f|$, we have, for each $\hat{z}_{j} \in \pi_{j} \Omega$,

$$
\log \left|f\left(\hat{z}_{j}\right)\right| \leqq \log ^{+}\left|f\left(\hat{z}_{j}\right)\right| \leqq \int_{U(a)} \log ^{+}|f(z)| d m\left(z_{j}\right)
$$

Hence integration over $\pi_{j} \Omega$ gives

$$
\begin{aligned}
\int_{\pi, \Omega} \log \left|f\left(\hat{z}_{j}\right)\right| d m\left(\hat{z}_{j}\right) & \leqq \int_{\pi, \Omega} \log ^{+}\left|f\left(\hat{z}_{j}\right)\right| d m\left(\hat{z}_{j}\right) \\
& \leqq \int_{\pi_{j} \Omega} d m\left(\hat{z}_{j}\right) \int_{U(a)} \log ^{+}|f(z)| d m\left(z_{j}\right) \\
& =\int_{\Omega} \log ^{+}|f(z)| d m(z)
\end{aligned}
$$


The first integral is $>-\infty$ by Theorem 5.2 of [1] and the last integral is $<+\infty$ by Lemma 3.2.

LEMMA 3.4. Let $f \in N(\Omega)$ and $1 \leqq j \leqq n+1$. If $f\left(\hat{z}_{j}\right) \not \equiv 0$, then with the notation of $\S 2$,

$$
\int_{\pi, \Omega} d H_{2 n}\left(\hat{z}_{j}\right) \int_{T} \log \left|f_{\hat{z}_{j}^{*}}^{*}(a w)\right| d m_{1}(w)<\infty .
$$

We give separate proofs for $\Omega=U^{n+1}$ and $\Omega=B_{n+1}$.

For $U^{n+1}$, we consider only the case $j=n+1$; the other cases can be treated similarly. For $w=\left(w_{1}, \ldots, w_{n}, w_{n+1}\right) \in T^{n+1}$, write $w^{\prime}=\left(w_{1}, \ldots, w_{n}\right)$. Since $\log |f|$ is $(n+1)$-subharmonic, we have

$$
\int_{T^{n}} d m\left(w^{\prime}\right) \int_{T} \log \left|f^{*}\left(r_{1} w_{1}, \ldots, r_{n} w_{n}, w_{n+1}\right)\right| d m_{1}\left(w_{n+1}\right) \leqq \int_{T^{n+1}} \log \left|f^{*}(w)\right| d m(w)
$$

for $0 \leqq r_{j}<1,1 \leqq j \leqq n$. The last integral is $\leqq\|f\|_{0}$ by Theorem 3.1. Multiply by $r_{1} d r_{1} \cdots r_{n} d r_{n}$ and integrate from 0 to 1 for each $r_{j}$. This gives

$$
\int_{U^{n}} d m\left(z^{\prime}\right) \int_{T} \log \left|f^{*}\left(z^{\prime}, w_{n+1}\right)\right| d m_{1}\left(w_{n+1}\right) \leqq 2^{-n}\|f\|_{0} .
$$

The integral is $\geqq \int_{U^{n}} \log \left|f\left(z^{\prime}, 0\right)\right| d m\left(z^{\prime}\right)>-\infty$ by Theorem 5.2 of [1].

For $B_{n+1}$, we fix $0 \leqq r<1$ and let $b=\left(r^{2}-\left\|\hat{z}_{j}\right\|^{2}\right)^{1 / 2}$. Then $b \rightarrow a$ as $r \rightarrow 1$.

By Corollary 4.2 below,

$$
\begin{aligned}
\int_{\pi, B_{n+1}(r)} d H_{2 n}\left(\hat{z}_{j}\right) \int_{T} r \log ^{+}\left|f_{\hat{z}_{j}}(b w)\right| d m_{1}(w) & =\frac{1}{2 \pi} \int_{\partial_{B_{n+1}(r)}} \log ^{+}|f(w)| d m(w) \\
& =\frac{r^{2 n+1}}{2 \pi} \int_{\partial_{B_{n+1}}} \log ^{+}|f(r w)| d m(w) \\
& \leqq \frac{r^{2 n+1}}{2 \pi}\|f\|_{0} .
\end{aligned}
$$

So

$$
\int_{\pi, B_{n+1}(r)} d H_{2 n}\left(\hat{z}_{j}\right) \int_{T} \log ^{+}\left|f_{\hat{z}_{j}}(b w)\right| d m_{1}(w) \leqq \frac{\|f\|_{0}}{2 \pi} .
$$

The integral $\varphi_{r}\left(\hat{z}_{j}\right)=\int_{T} \log ^{+}\left|f_{\hat{z}_{j}}(b w)\right| d m_{1}(w)$ is an increasing function of $r$. Its limit $\varphi\left(z_{j}\right)$ is integrable over $\pi_{j} B_{n+1}$. Hence $\varphi\left(\hat{z}_{j}\right)<\infty$ for almost all $\hat{z}_{j}$. For each such $\hat{z}_{j}, f_{\varepsilon_{j}}^{*}(a w)$ exists for almost all $w \in T$. Furthermore,

$$
\begin{array}{rl}
\int_{\pi_{j} B_{n+1}} & d H_{2 n}\left(\hat{z}_{j}\right) \int_{T} \log \left|f\left(a_{z_{j}}^{*} w\right)\right| d m_{1}(w) \\
& \leqq \int_{\pi_{j} B_{n+1}} d H_{2 n}\left(\hat{z}_{j}\right) \int_{T} \log ^{+}\left|f_{z j}^{*}(a w)\right| d m_{1}(w) \leqq \frac{\|f\|_{0}}{2 \pi} .
\end{array}
$$

\section{A lemma on measures.}

LeMma 4.1. Let $V$ be an open subset of $\boldsymbol{R}^{k}$ and let $\Phi: V \rightarrow M$ be a $C^{1}$ map from 
$V$ onto a subset $M$ of $\boldsymbol{R}^{n}$. Let $f$ be a Borel measurable function on $M$. Then $f(y) H_{0}\left\{V \cap \Phi^{-1}(y)\right\} \in L^{1}(M)$ if and only if $f(\Phi(x)) J \Phi(x) \in L^{1}(V)$ and then

$$
\int_{M} f(y) H_{0}\left\{V \cap \Phi^{-1}(y)\right\} d H_{k}(y)=\int_{V} f(\Phi(x)) J \Phi(x) d H_{k}(x)
$$

where $J \Phi$ is the Jacobian of $\Phi$.

Proof. When $f$ is the characteristic function of a Borel set in $M$, formula (4.1) was proved by Federer (see [1, Theorem 3.1]). The general case follows by the usual extension process from characteristic functions to $L^{1}$ functions.

Corollary 4.2. For $k \geqq 2$, let $B_{k}$ be the unit ball in $\boldsymbol{R}^{k}, S^{k-1}=\partial B_{k}$. Write points of $S^{k-1}$ in the form $(x, \theta)=(x, a \cos \theta, a \sin \theta)$ where $x \in B_{k-2},-\pi \leqq \theta<\pi$ and $a^{2}=1-\|x\|^{2}$. Then $\int_{S^{k-1}} f(w) d m(w)=\int_{B_{k-2}} d m(x) \int_{-\pi}^{\pi} f(x, \theta) d \theta$ for all $f \in L^{1}\left(S^{k-1}\right)$.

Proof. Put $V=B_{k-2} \times(-\pi, \pi), \Phi(x, \theta)=(x, a \cos \theta, a \sin \theta)$ in Lemma 4.1. Then $\Phi$ is $1-1$ on $V$ and $J \Phi\left(x_{1}, \theta\right)=1$. The image of $\Phi$ is all of $S^{k-1}$ minus a set of measure zero. The corollary follows by applying Fubini's theorem to $V$.

Added in Proof. Professor E. L. Stout has obtained similar but more general results as our Theorem 1.1, and also a different proof of Corollary 4.2.

\section{REFERENCES}

1. P. S. Chee, The Blaschke condition for bounded holomorphic functions, Trans. Amer. Math. Soc. 148 (1970), 249-263.

2. W. Rudin, Function theory in polydiscs, Math. Lecture Notes, Benjamin, New York, 1969.

3. E. C. Titchmarsh, The theory of functions, 2nd ed., Oxford Univ. Press, Oxford, 1939.

UNIVERSITY OF MALAYA,

Kuala Lumpur, Malaysia 